\title{
Análise de Aplicativos para Pessoas Analfabetas: Quais são as suas Características?
}

\author{
Alexander Soledade ${ }^{1}$, Geisy Anny Venancio ${ }^{1}$, Priscila Fernandes ${ }^{1}$, \\ Vitor Bremgartner $^{1}$ e Luis Rivero ${ }^{2}$
}

\author{
${ }^{1}$ Instituto Federal de Educação, Ciências e Tecnologia e do Amazonas (IFAM) \\ Manaus, AM - Brasil \\ ${ }^{2}$ Programa de Pós-Graduação em Ciência da Computação (PPGCC), \\ Universidade Federal do Maranhão (UFMA) - São Luís, MA - Brasil \\ \{2021000964, geisy.venancio, priscila.fernandes, \\ vitorbref\}@ifam.edu.br, luisrivero@nca.ufma.br
}

\begin{abstract}
As technology is developed, access to information becomes increasingly democratic. However, some social chasms persist and among them there is illiteracy. In order to democratize access to technology, tools need to be developed, also thinking about rescuing those on the margins of Society. Thus, the aim of this paper was to map the availability of targeted applications to facilitate the learning of illiterate people from groups of children, young people and adults. Despite the great technological leverage, it is necessary to ensure that less favored classes have access to low-cost tools, forcing developers to create supported software on any phone.
\end{abstract}

Resumo. À medida que a tecnologia é desenvolvida, o acesso à informação torna-se cada vez mais democrático. No entanto, alguns abismos sociais persistem e dentre eles existe o analfabetismo. Para que haja a democratização do acesso à tecnologia, ferramentas precisam ser desenvolvidas pensando também em resgatar quem está à margem da sociedade. Dessa forma, o objetivo deste artigo foi realizar um mapeamento da disponibilidade de aplicativos direcionados para facilitar a aprendizagem de pessoas analfabetas de grupos de crianças, jovens e adultos. Apesar da grande alavancagem tecnológica, é preciso certificar que classes menos favorecidas tenham acesso às ferramentas de custo baixo, obrigando aos desenvolvedores a criarem softwares suportados em qualquer aparelho telefônico.

\section{Introdução}

Em meio aos avanços tecnológicos evidenciados nos últimos anos, a popularização digital promove inúmeras possibilidades para os usuários, de forma que sejam construídas práticas sociais e tecnológicas para a comunicação virtual. A internet torna-se cada vez mais diversificada e ampla, presente em qualquer ambiente do dia a dia dos indivíduos, sejam em praças, bancos e quaisquer outros espaços públicos. Assim, esta é principalmente utilizada para o desenvolvimento dos usuários que buscam por alternativas de informações rápidas e eficientes, por meio de sites de notícias, redes sociais e jornais virtuais, de modo que haja a identificação do processo de informação, bem como da estruturação do próprio conhecimento [VILAÇA and Araujo 2016]. 
De certo, as tecnologias utilizadas para o beneficiamento dos usuários, configuram-se como uma nova forma de desenvolvimento da sociedade, permitindo assim, a melhoria dos aspectos comunicativos, além dos meios sociais, políticos e econômicos. Usada como ferramenta para auxiliar as etapas de conhecimento, a internet promove a agilidade de informações, como por exemplo, em situações que almejam o encontro de pessoas, lugares, e ainda, a possibilidade de compras que cresce a cada ano, a partir de inúmeras plataformas de acesso [OLIVEIRA and MORAES 2013].

Em paralelo a todo o crescimento tecnológico, muitos abismos sociais precisam ser sanados, sendo o analfabetismo um deles. Segundo o Instituto Brasileiro de Geografia e Estatística [Bruns and da Cunha Nunes 2021], 11 milhões de brasileiros não sabem ler nem escrever, e em um cenário otimista, até 2024 o Plano Nacional de Educação pretende erradicar o analfabetismo no Brasil, no entanto, com o advento da pandemia muitas escolas suspenderam aulas presenciais e as saídas tecnológicas precisam ser desenvolvidas.

Atualmente, a criação de aplicativos para o combate ao analfabetismo está sendo desenvolvida como uma maneira de minimizar os impactos causados pela precariedade social [da Fonseca et al. 2018]. Os meios de comunicação voltados para o processo de aprendizagem tornam-se cada vez mais amplos, promovendo a democratização de acessos básicos, como a leitura e a escrita.

Apesar da ampla diversidade de aplicativos facilitadores da comunicação, como por exemplo, WhatsApp, que possui funções diversas de envio de mensagens, seja por vídeo, áudio ou imagem, o foco foi avaliar apenas aplicativos que auxiliam na aprendizagem de indivíduos que estão em situações de analfabetismo e buscam por alternativas para desenvolver a leitura, escrita e compreensão de textos. Dessa forma, o objetivo deste artigo consistiu na execução de um mapeamento da disponibilidade de aplicativos direcionados para facilitar a aprendizagem de pessoas analfabetas, de modo a analisar e comparar suas características, presentes em canais de distribuição como a Play Store.

\section{Método}

A pesquisa possui cunho qualitativo de caráter bibliográfico, de modo a auxiliar a disponibilidade de informações sobre o assunto previsto na literatura acadêmica com a finalidade de aprofundá-lo para posterior beneficiamento dos leitores e público-alvo. O estudo foi realizado no ambiente virtual, em plataformas digitais como: SciELO, Periódicos CAPES, ERIC e Google Acadêmico.

A população estudada considerou seis aplicativos existentes em canais de distribuição como a Play Store, considerando três públicos de indivíduos analfabetos ou semianalfabetos, sendo eles crianças, jovens e adultos. Os seis aplicativos representam um todo do que há disponível para atendê-los. Assim, as amostras conseguem abranger todas as características que atendem o analfabetismo, e cabe a um próximo estudo avaliar aplicativos por públicos, ou seja, somente para crianças, jovens ou adultos.

Os procedimentos metodológicos consistiram em três etapas principais: Levantamento bibliográfico através de periódicos e teses; Coleta e análises de informações para o desenvolvimento de um banco de dados; e Análise dos aplicativos direcionados para analfabetos, através dos materiais selecionados. Foi utilizada uma análise comparativa, utilizando a técnica de análise de características [Kitchenham 1997] que teve como objetivo analisar os principais programas e seus recursos disponíveis. 


\subsection{Levantamento Bibliográfico}

O levantamento de dados da pesquisa bibliográfica foi realizado por palavras- chaves que se adequassem ao tema referentes às publicações realizadas por autores diversos, como: Analfabetismo; Aplicativos; Desenvolvimento tecnológico. Foi usado no intuito de obter informações pertinentes ao assunto um artigo onde o aplicativo palma é analisado [da Fonseca et al. 2018].

\subsection{Coleta e Análise de Informações}

A coleta das informações consistiu na pesquisa dos documentos e armazenamento em aplicativo de bloco de notas como o OneNote ${ }^{\circledR}$ para a documentação de todas as observações relevantes da pesquisa. A análise dos dados foi realizada por meio de avaliação de materiais e documentos já existentes disponível em arquivos digitais. Este artigo se diferencia dos demais pois trata de vários aplicativos, para assim gerar uma conclusão mais abrangente para o leitor.

\subsection{Análise de aplicativos para pessoas analfabetas}

A investigação dos aplicativos consistiu na pesquisa por palavras-chaves como: Analfabetismo e Alfabetismo. Os aplicativos avaliados foram:

a. Palma ${ }^{1}$ (Programa de Alfabetização na Língua Materna): O Palma, desenvolvido na cidade de Itatiba no interior de São Paulo, é um programa que inclui vários aplicativos com o objetivo de estimular e complementar, por meio digital, os acessos básicos como ler, escrever e compreender textos. Ele envolve uma série de combinações de sons, letras, imagens e envio de dados que possui cinco níveis de dificuldade: alfabeto, sílabas simples, sílabas complexas, universo vocabular e leitura e compreensão de textos.

b. $\mathrm{ABC}^{2}$ : O programa, criado pelas estudantes do curso de Ciências Matemáticas e de Computação (ICMC) da USP, em São Carlos é direcionado para pessoas que podem ler sentenças curtas, escrever o próprio nome, mas são incapazes de ler livros - conhecidos como analfabetos funcionais. A solução também poderá ser útil para os que são completamente analfabetos, ou seja, não conseguem ler ou escrever nem uma palavra. Nesse caso, eles precisarão de apoio. O projeto $\mathrm{ABC}$, foi moldado de forma a ajudar na alfabetização por meio de vídeos e dicas. Será possível também avaliar o progresso do aprendizado respondendo as questões e realizando exercícios. A proposta é que os usuários possam aprender, escolhendo as lições segundo seus temas de interesse. O novo desafio em 2021 é colocar o projeto em prática e disponibilizá-los em plataformas. Para isso, as estudantes estão buscando trocar experiências com quem já desenvolveu aplicativos e com especialistas na área de educação.

c. EduEdu ${ }^{3}: \mathrm{O}$ aplicativo EduEdu permite que a criança desenvolva atividades personalizadas, de modo a promover o seu sucesso escolar, acompanhando a evolução e monitorando seu progresso na construção de novas atividades. Possui inúmeros jogos que incentivam a diversão, o dinamismo, além de apresentar músicas e textos diversificados.

d. Ler e Contar ${ }^{4}$ : É um aplicativo para o ensino da leitura, que pode ser utilizado

\footnotetext{
${ }^{1}$ Palma: https://www.palmaescola.com.br/

${ }^{2} \mathrm{ABC}$ : http://www.saocarlos.usp.br/aplicativo-para-alfabetizacao-equipe-de-alunas-da-usp-e-premiada -em-desafio-internacional/

${ }^{3}$ EduEdu: https://www.eduedu.com.br/

${ }^{4}$ Ler e Contar: https://play.google.com/store/apps/details?id=com.appsbergman.silabando
} 
por crianças maiores e menores que estão em fase de alfabetização e aprendizagem de matemática. Além de letras e números, a plataforma traz formas, animais, cores, instrumentos musicais e Libras (língua brasileira de sinais).

Cada opção do menu tem uma série de atividades diferentes para ajudar no aprendizado, seja colorir um animal de estimação, adivinhar a primeira letra de uma palavra ou fazer com que sua mão use pontos do alfabeto. Os adultos podem escolher português, espanhol e inglês, o que também ajuda no ensino de outros idiomas.

e. Silabando 5 : O Silabando ensina a formar, separar e contar sílabas. Elas estão divididas no app em dois grupos, simples e complexas, que devem ser selecionadas de acordo com o estágio de alfabetização da criança.

Os grupos trazem seis tipos de exercício: apresentação e formação de sílabas; identificação da parte que falta para formar a palavra; acertar a sílaba que é pedida pelo app; ler palavras com a separação silábica feita e, por fim, contar o número de sílabas de uma palavra.

f. Playkids ${ }^{6}$ : Não é um aplicativo de leitura específico. A plataforma inclui conteúdos educativos e de entretenimento, como desenhos animados como Turma da Mônica, Masha e o Urso e O Show da Luna, além de contos e jogos infantis de Natal.

Na parte dedicada à alfabetização, a plataforma possui um espaço "mundo da leitura e aprendizagem em casa", que traz conteúdos em português e outros temas. O Playkids é um aplicativo pago (R\$ 29,90 por mês ou R\$ 199,90 por ano), mas você pode experimentá-lo gratuitamente por três dias.

\section{Resultado e Discussão}

A Tabela 1 a seguir apresenta um comparativo dos aplicativos avaliados e suas principais características. Por sua vez, a Figura 1 apresenta prints de tela dos aplicativos analisados. Nesse contexto, as características abordadas na tabela são: Tamanho do arquivo, versão gratuita, Modos offline e online, público-alvo, análise do conhecimento do usuário, possui avaliação de aprendizagem, disponibilidade em plataformas e analfabetos totais e funcionais.

O tamanho do arquivo refere-se à quantidade de megabytes (MB) do arquivo para download e instalação do programa. Por sua vez, a versão gratuita indica se os aplicativos possuem licença ou não para serem utilizados; Os modos online e offline dizem respeito aos aplicativos que necessitam estar conectados à internet para o seu uso; O público-alvo indica para qual faixa etária eles são direcionados; A análise do conhecimento do usuário permite realizar a identificação de informações a respeito do status do indivíduo que utiliza o aplicativo, ou seja, em qual etapa da alfabetização eles se encontram; Avaliação do conhecimento permite que o programa realize uma análise ao final de cada atividade ou etapa para identificar o desempenho de cada usuário; a disponibilidade em plataformas significa se o aplicativo está disponível para o acesso, seja na Play Store ou Apple Store.

Já as características relacionadas aos analfabetos totais e funcionais evidenciam se os usuários não sabem ler e escrever de forma total (analfabetos totais) ou se eles sabem

\footnotetext{
${ }^{5}$ Silabando: https://play.google.com/store/apps/details?id=com.bergman.lerecontar

${ }^{6}$ Playkids: https://playkids.com/
} 
Tabela 1. Resumo da análise comparativa dos aplicativos para analfabetos

\begin{tabular}{|c|c|c|c|c|c|c|}
\hline \multirow[b]{2}{*}{ Características } & \multicolumn{6}{|c|}{ Aplicativos } \\
\hline & 㖒 & 总 & 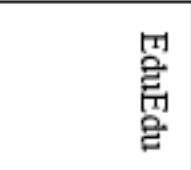 & 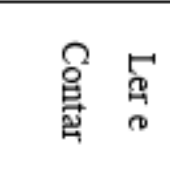 & 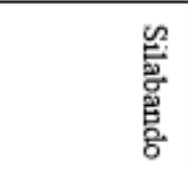 & $\begin{array}{l}\text { 禀 } \\
\text { : } \\
\text { : }\end{array}$ \\
\hline $\begin{array}{c}\text { Tamanho do } \\
\text { arquivo }\end{array}$ & $9,1 \mathrm{MB}$ & $\begin{array}{c}\text { Não } \\
\text { informado }\end{array}$ & $77 \mathrm{MB}$ & $40 \mathrm{MB}$ & $24 \mathrm{MB}$ & $125 \mathrm{MB}$ \\
\hline Versão gratuita & Não & Sim & Sim & Sim & Sim & $\operatorname{Sim}$ \\
\hline $\begin{array}{c}\text { Modos offline } \mathrm{e} \\
\text { online }\end{array}$ & Online & Offline & Online & Online & Offline & Online \\
\hline Público-alvo & $\begin{array}{l}\text { Jovens e } \\
\text { Adultos }\end{array}$ & Adultos & Crianças & Crianças & $\begin{array}{l}\text { Jovens e } \\
\text { Adultos }\end{array}$ & $\begin{array}{l}\text { Jovens e } \\
\text { Adultos }\end{array}$ \\
\hline $\begin{array}{l}\text { Análise do } \\
\text { conhecimento } \\
\text { do usuário }\end{array}$ & Não & Sim & Sim & Não & Não & Sim \\
\hline $\begin{array}{c}\text { Possui } \\
\text { avaliação de } \\
\text { aprendizagem }\end{array}$ & Sim & Sim & Sim & Não & Sim & Sim \\
\hline $\begin{array}{l}\text { Disponibilidade } \\
\text { em plataformas }\end{array}$ & $\begin{array}{l}\text { Play } \\
\text { Store }\end{array}$ & $\begin{array}{l}\text { Ainda não } \\
\text { disponivel }\end{array}$ & Play Store & $\begin{array}{l}\text { Play } \\
\text { Store }\end{array}$ & Play Store & $\begin{array}{l}\text { Play } \\
\text { Store }\end{array}$ \\
\hline $\begin{array}{l}\text { Analfabetos } \\
\text { totais e } \\
\text { funcionais }\end{array}$ & $\begin{array}{c}\text { Totais e } \\
\text { funcionais }\end{array}$ & Funcionais & Funcionais & $\begin{array}{l}\text { Totais e } \\
\text { funcionais }\end{array}$ & Funcionais & $\begin{array}{c}\text { Totais e } \\
\text { funcionais }\end{array}$ \\
\hline
\end{tabular}




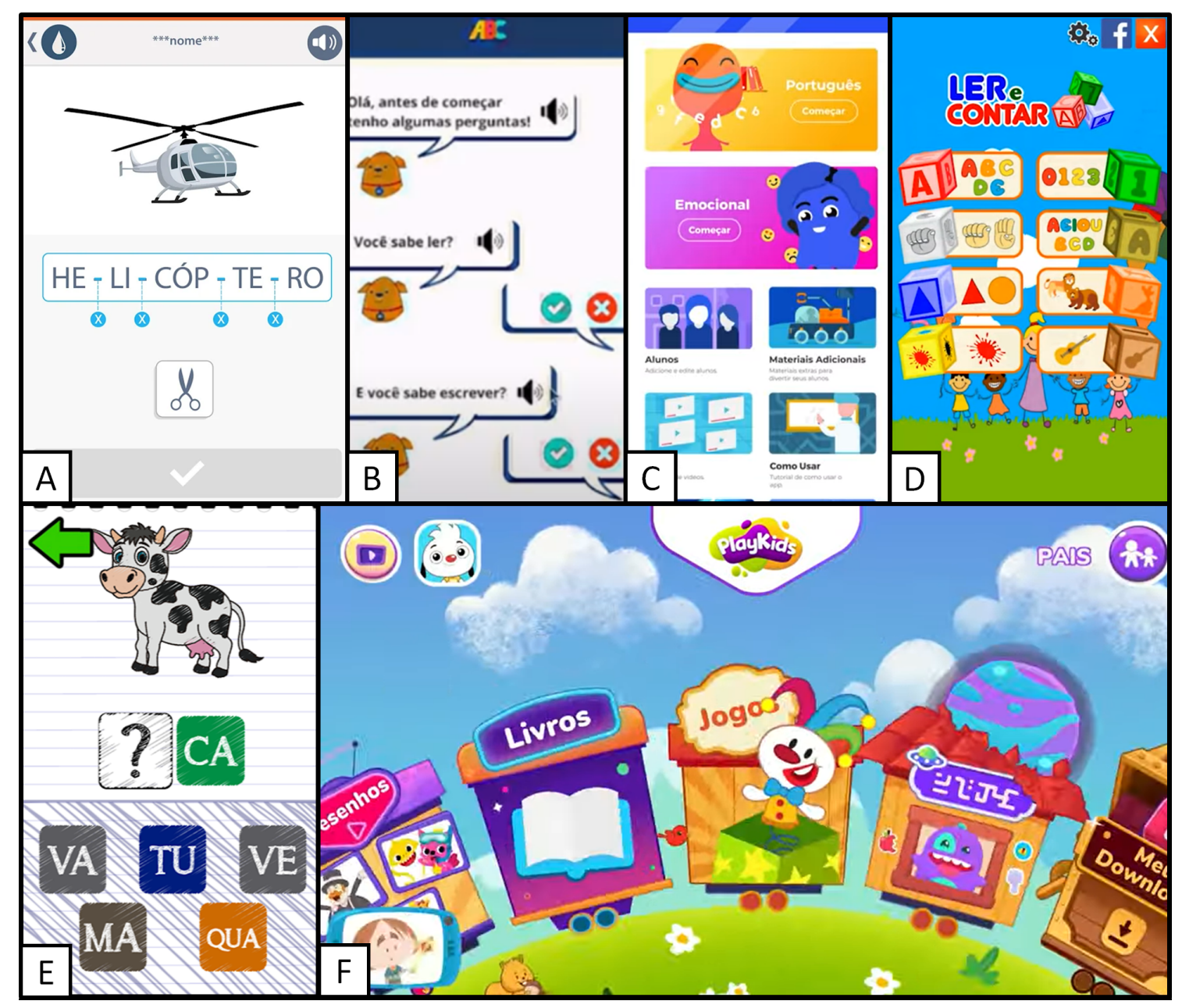

Figura 1. Prinst de telas dos aplicativos analisados: (A) Palma, (B) ABC, (c) EduEdu, (D) Ler e Contar, (E) Silabando e (F) Playkids.

ler e escrever, mas são incapazes de interpretar o que lê ou de utilizar essas contribuições em atividades cotidianas [Winck 2019].

De acordo com a Tabela 1, a característica de tamanho do arquivo pode estabelecer relações entre a capacidade de armazenamento do mesmo com o valor do aparelho celular que os usuários utilizam para acessar o aplicativo. Assim, uma quantidade menor de $\mathrm{KB}$ ou MB influenciam diretamente no poder de compra do smartphone devido à sua precificação. Dessa forma, o aplicativo Palma obtém uma quantidade de 9,1 MB, estabelecendo menor valor em relação aos demais. $\mathrm{O}$ aplicativo $\mathrm{ABC}$, por sua vez, não possui tal informação, devido a este ainda não ser apresentado disponível em plataformas digitais, o projeto está em processo de desenvolvimento e ainda não se tem o produto final, mas a ideia está sendo concluída e finalizada.

Em relação à versão gratuita, o aplicativo EduEdu se caracteriza como um meio gratuito para as crianças desenvolverem ou construírem suas habilidades. O aplicativo $\mathrm{ABC}$ apesar de ainda não se encontrar disponível, reforça a ideia de que com patrocínios, este será acessível para todos os usuários sem a necessidade de pagamento ou compra de licença. Os demais aplicativos são gratuitos com excessão do Palma. 
Para os modos a serem utilizados, um dos únicos que apresentaram características em modo offline foi o ABC e o Silabando, sendo que no ano de 2019 no Brasil, aproximadamente 120 milhões de brasileiros possuem acesso à Internet, gerando um total de $67 \%$ da população, o que diz respeito às classes $\mathrm{A}$ e B, tem-se que $90 \%$ das pessoas estão conectadas no dia a dia [Diniz et al. 2020]. No entanto, para as classes D e E, apenas $42 \%$ dos indivíduos possuem acesso à internet. Esse dado demonstra que para um analfabeto que não possui internet de qualidade, não adquire ferramentas suficientes para o seu desenvolvimento e estudo, mesmo com a disponibilidade de aplicativos.

O público-alvo do aplicativo Palma são jovens e adultos, abrangendo analfabetos totais e funcionais. O ABC abrange usuários adultos e o EduEdu, apenas crianças e ambos os aplicativos envolvem analfabetos funcionais. Em relação à característica do nivelamento do conhecimento, apenas o aplicativo Palma não possui tal propriedade. No entanto esta é de extrema importância, pois o objetivo é colocar o usuário em um nível que não seja fácil demais, contenha as dúvidas e gere aprendizado [de Sousa et al. 2020]. Escolhe-se começar no básico ou pulá-lo com o teste de nivelamento, otimizando seu tempo com avaliações periódicas.

A avaliação da aprendizagem está presente em quase todos os aplicativos avaliados, estando ausente apenas no aplicativo Ler e Contar. Esta é importante, pois indicará o nível dos usuários referentes as etapas de cada atividade ou prova desenvolvida. A análise, segundo Vargas (2018), contribui positivamente para o processo de educação, ensino e aprendizagem de maneira que, com o uso de aplicativos, a inovação e o dinamismo chamem a atenção dos usuários de forma estimuladora.

Em relação à disponibilidade de plataforma, o aplicativo $\mathrm{ABC}$ ainda não está disponível em nenhum meio digital, de forma que, como foi citado anteriormente, o projeto encontra-se em nível de finalização. Para os aplicativos Palma e EduEdu, ambos estão disponíveis apenas na Play Store. Este fator corrobora para que haja uma limitação destes usuários apenas para o seu uso em smartfones que contenha tal canal de distribuição.

Dessa forma, os meios para minimizar o analfabetismo estão sendo desenvolvidos constantemente no Brasil, de forma que usuários de aplicativos que os utilizam para este fim, possam se tornar cada vez mais proficientes em sua própria língua, com o objetivo de eliminar as dificuldades enfrentadas pela leitura, escrita e compreensão textual, permitindo assim, a ampliação de conhecimentos e promovendo uma melhor interação no dia a dia através do domínio de linguagens verbais [Nogueira and Challegre 2012].

\section{Considerações finais}

Com o desenvolvimento da pesquisa, constatou-se que aplicativos devem ser acessíveis em relação às características operacionais, sendo elas simples e que sejam suportadas em aparelhos com valores acessíveis às classes mais baixas. O acesso à internet ainda hoje é uma limitação para a utilização das ferramentas. Sendo assim, desenvolver aplicativos que possam ser utilizados em modo offline, sana parte do problema.

Por fim, o estudo comparativo proporcionou o desenvolvimento de um breve resumo entre ferramentas que possuem o objetivo de auxiliar na alfabetização dos indivíduos. Abre-se o leque para estudos relacionados às questões sociais e de acessibilidade à tecnologia, permitindo futuras pesquisas vinculadas não somente ao nicho tecnológico, mas também com o objetivo de sanar grandes lacunas sociais. Adicionalmente, 
pretende-se executar estudos experimentais do ponto de vista dos usuários, aplicando questionários consolidados com o do modelo de aceitação de tecnologia (TAM) e seus derivados [Dasgupta et al. 2002] para avaliar a percepção dos usuários sobre os aplicativos propostos. Espera-se, com este trabalho, apoiar o combate ao analfabetismo, fornecendo sistemas no contexto do ensino.

\section{Agradecimentos}

Parte dos resultados apresentados neste trabalho foram obtidos através do PROJETO ARANOUÁ, financiado pela Samsung Eletrônica da Amazônia Ltda., com recursos previstos na Lei Federal nº $8.387 / 1991$.

\section{Referências}

Bruns, J. P. and da Cunha Nunes, C. (2021). Implicações da implementação do programa mais alfabetização: reflexões a partir da gestão escolar. Série-Estudos-Periódico do Programa de Pós-Graduação em Educação da UCDB, pages 243-262.

da Fonseca, A. G. M. F. et al. (2018). Programa palma: Dispositivos móveis e aplicativo como ferramenta para alfabetização. Aturá-Revista Pan-Amazônica de Comunicação, 2(1):250-274.

Dasgupta, S., Granger, M., and McGarry, N. (2002). User acceptance of e-collaboration technology: An extension of the technology acceptance model. Group Decision and Negotiation, 11(2):87-100.

de Sousa, K. F., de Lima Neto, F. R., and Araújo, N. M. S. (2020). Duolingo for schools: avaliando o uso de uma ferramenta com traços de gamificação no ifce. LínguaTec, 5(2):169-187.

Diniz, J. L., Moreira, A. C. A., Teixeira, I. X., Azevedo, S. G. V., Freitas, C. A. S. L., and Maranguape, I. C. (2020). Digital inclusion and internet use among older adults in brazil: a cross-sectional study. Revista Brasileira de Enfermagem, 73.

Kitchenham, B. A. (1997). Evaluating software engineering methods and tools, part 7: planning feature analysis evaluation. ACM SIGSOFT software engineering Notes, 22(4):21-24.

Nogueira, N. and Challegre, V. (2012). Impacto das ferramentas assistivas na educação de analfabetos funcionais. Conferencias LACLO, 3(1).

OLIVEIRA, F. N. d. and MORAES, D. A. F. d. (2013). A utilização da tecnologia e da internet no processo de ensino e aprendizagem da educação superior: um relato de experiência. Jornada da Didática, 2.

Vargas, D. d. (2018). O processo de aprendizagem e avaliação através de quiz.

VILAÇA, M. L. C. and Araujo, E. d. (2016). Tecnologia, sociedade e educação na era digital. Duque de Caxias: UNIGRANRIO.

Winck, F. (2019). Analfabetismo funcional entre vestibulandos dos cursos de graduação em letras, história e jornalismo. 